\title{
EFECTO IN VITRO DE LA SOLUCIÓN DE Caesalpinia espinosa (TARA) AL 60\%, E HIDRÓXIDO DE CALCIO Y GLUCONATO DE CLOREXHIDINA AL 2\% EN EL HALO INHIBITORIO MICROBIANO DE Enterococcus faecalis
}

\author{
IN VITRO EFFECT OF A SOLUTION OF Caesalpinia spinosa AT 60\%, AND \\ CALCIUM HYDROXIDE AND CHLORHEXIDINE GLUCONATE AT 2\% IN \\ THE MICROBIAL INHIBITION HALO OF Enterococcus faecalis
}

${ }^{1}$ Juan Guillermo Bornaz Acosta; ${ }^{2}$ Vanessa Lisethe Bornaz Arenas; ${ }^{3}$ Milagros Catherine Bornaz Arenas

\section{RESUMEN}

La presente investigación tuvo como objetivo determinar el halo inhibitorio de la Caesalpinia espinosa (Tara), de Hidróxido de Calcio y de Gluconato de clorhexidina al $2 \%$ sobre la cepa de Enterococcus faecalis, bacteria bastante conocida por provocar elevado porcentaje de fracasos en endodoncia. El procedimiento consistió en sembrar la cepa Enterococcus faecalis (ATCC 29212) en 16 placas con Agar Cerebro Corazón, las cuales fueron divididas en 3 unidades por placa, evaluándose así 48 unidades de estudio, 24 unidades para el grupo experimental 1 ( Caesalpinia espinosa al $60 \%$ ) y 24 para el grupo experimental 2 (Hidróxido de Calcio y Gluconato de clorhexidina al $2 \%$ ). Se adicionaron 4 placas petri, 3 para el control positivo (Amoxicilina - Acido clavulánico) y 1 placa Petri para el control negativo (Suero fisiológico). Se colocaron sensidiscos embebidos con la solución de Caesalpinia espinosa y se hizo pozos de $5 \mathrm{~mm}$ para el hidróxido de calcio y clorhexidina al $2 \%$, posteriormente las placas fueron incubadas en cámara de anaerobiosis a una temperatura de $37{ }^{\circ} \mathrm{C}$, tomándose medidas de halo inhibitorio expresado en milimetros a las $24,48,72$ horas y 7 días. Los resultados obtenidos fueron sistematizados, luego se aplicó el Estadístico T de Student $(\mathrm{p}<0,05)$ indicando que el promedio del halo inhibitorio formado por la Caesalpinia espinosa fue mayor que el halo inhibitorio formado por el Hidróxido de Calcio, habia diferencia estadisticamente significativa entre los datos obtenidos entre ambos grupos experimentales. En conclusión, la Caesalpinia espinosa demostró tener efecto antimicrobiano frente a la presencia de Enterococcus faecalis. formando halos de diferentes diámetros en las 4 tomas de medidas que se realizó en este estudio.

Palabras clave: Antimicrobiano, fracaso endodóntico, Enterococcus faecalis, Caesalpinia espinosa, hidróxido de calcio y gluconato de clorhexidina

\section{ABSTRACT}

The present study aimed to determine the inhibitory halo Caesalpinia espinosa (Tara) calcium hydroxide and chlorhexidine gluconate $2 \%$ on Enterococcus faecalis strain, well known for causing bacteria large percentage of failures in endodontics. The procedure consisted in sowing Enterococcus faecalis strain (ATCC 29212) in 16 Brain Heart Agar plates, which were divided into 3 units per plate, evaluated and 48 studio units, 24 units for the experimental group 1 (Caesalpinia espinosa $60 \%$ ) and 24 for the experimental group 2 (calcium hydroxide and chlorhexidine gluconate $2 \%$ ). Petri plates were added in April, 3 for the positive control (amoxicillin - clavulanic acid) and a Petri dish for the negative control. Caron was placed sensidises embedded with prickly caesalpinia solution was $5 \mathrm{~mm}$ wells calcium hydroxide, posteriormentes the plates were incubated in anaerobic chamber at a temperature of $37{ }^{\circ} \mathrm{C}$, taking inhibitory halo measures expressed in millimeters to $24,48,72$ hours and 7 days. The results were systematized then applied the Student's T-statistic $(\mathrm{p}<0,05)$ indicating that the mean inhibitory halo formed by the Caesalpinia espinosa was greater than the inhibitory halo formed by Calcium Hydroxide, there was statistically significant difference between the data obtained between both experimental groups. In conclusion, the Caesalpinia espinosa proved to have antimicrobial effect against the presence of Enterococcus faecalis, forming halos of different diameters on 4 outlets of measures made in this study.

Keywords: Antimicrobial, endodontic failure, Enterococcus faecalis, Caesalpinia espinosa, calcium hydroxide and chlorhexidine gluconate

\section{INTRODUCCIÓN}

Unas de las enfermedades más comunes en la humanidad es la caries y ésta tiene como consecuencia la afectación de los tejidos pulpares, en el caso de estar en esta condición se deberá realizar una endodoncia, especialidad de la odontología utilizada para la remoción del tejido pulpar y así remover el daño ( 1 y 2). Existen medicamentos que se utilizan en esta terapia, algunos de estos medicamentos o la mayor parte de ellos fracasan cuando se enfrentan al Enterococcus faecalis (3 y 4). En la actualidad, según estudios previos, una de las bacterias más persistentes responsable de

Doctor en Ciencias en la especialidad de Biología. Facultad de Ciencias de la Salud de la Universidad Nacional Jorge Basadre Grohmann. Tacna-Perú Doctora en Odontología, Magister en Salud Pública, Cirujano Dentista. Facultad de Ciencias de la Salud de la Universidad Alas Peruanas. Arequipa-Perú. Cirujano Dentista. Essalud Tacna. Tacna-Perú. 


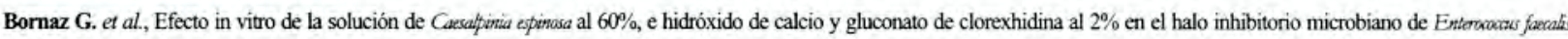

los fracasos endodónticos es el Enterococcus faecalis siendo esta cepa resistente a la mayoría de Sustancias utilizadas en Endodoncia (4 y 5).

En la actualidad, se cursa la era de la fotoquímica, y debemos de hacer los esfuerzos para que los productos naturales que posee nuestro país sean utilizados en provecho de nuestra población. Debido a que la Caesalpinia Espinosa, es un producto natural, no solo será capaz de eliminar los microorganismos, por su poder antimicrobiano demostrado en otros estudios, sino que también ayudará a lá mejor cicatrización, propiedad que también ha sido demostrada en estudios preliminares, además de observarse menor cantidad de efectos adversos, por tratarse de una sustancia de origen natural (7).

A pesar que estamos en una Era Odontológica Preventiva y que la profesión odontológica busca reducir los índices de caries y reducir los índices de higiene oral usando métodos, estrategias y programas preventivos de caries. Estas medidas tendrán su impacto en el futuro mientras que los procedimientos clínicos resuelven el daño que ya está presente, por lo tanto debería tomarse importancia a la necesidad de conservar las piezas ya afectadas por caries, eliminando los microorganismos más agresivos presentes en piezas necrosadas y poder realizar un tratamiento endodóntico que tenga éxito al $100 \%(7,8$ y 9$)$.

Se considera importante que se realicen estudios para determinar el efecto antimicrobiano de sustancias alternativas a las que ya existen, siendo las sustancias naturales las más indicadas, debido a que son mejor aceptadas por el organismo presentando menor cantidad de efectos colaterales, siendo éste el objetivo del estudio, determinar el efecto antimicrobiano de una sustancia extraida de un producto natural como es la Caesalpinia espinosa.

\section{MATERIAL Y METODOS}

\section{Obtención del extracto de Caesalpinia espinosa.}

Vainas secas de Caesalpinia espinosa fueron recolectadas y se procedió a separar las semillas de las vainas, posteriormente se molió las vainas obteniéndose un polvo fino, el cual se mezcló con alcohol de 96 grados en recipientes color caramelo, por 14 dias, durante los cuales se procedió a agitar por un lapso de 30 segundos diarios para homogenizar la solución. Se procedió a filtrar la solución con papel filtro y luego la solución obtenida se colocó en baño maría para vaporizar el alcohol. Posteriormente se colocó la sustancia obtenida en un recipiente adecuado para su conservación $(10)$.

\section{Preparación de los medios de cultivo y siembra.}

Se empleó la cepa Enterococcus faecalis (ATCC 29212) el cual fue reactivada incubándola en medio infusión Cerebro Corazón (BHI) por 24 horas a $37^{\circ} \mathrm{C}$, después de este procedimiento, la concentración celular fue medida por medio de espectrofotometría según la escala de 0,5 de $\mathrm{Mc}$ Farland. El medio de cultivo Agar Cerebro Corazón (BHA) se preparó, se esterilizó y puso en baño maría hasta su uso; se procedió a verter la concentración bacteriana de Cepa de Enterococcus faecalis en el Agar Cerebro Corazón, para luego repartir el medio en las placas petri previamente esterilizadas a razón de un espesor de $4 \mathrm{~mm}$ por placa dentro de la cámara de seguridad biológica, siguiendo los parámetros de bioseguridad (11).

Se dejó solidificar a temperatura de medio ambiente por 15 minutos, se rotuló las placas en la parte posterior con el nombre de la sustancia a investigar.

Una vez que el agar solidificó se procedió a colocar sensidiscos de $5 \mathrm{~mm}$ en tres puntos separados en la placa petri, con 30 microlitros con la sustancia de Caesalpinia espinosa al $60 \%$ y el suero fisiológico y se realizó pozos de 5 mm en tres puntos separados para colocar la pasta de Hidróxido de calcio + Gluconato de clorhexidina, y la pasta de Amoxicilina + Acido Clavulánico. A razón de 24 muestras para Grupo Experimental 1: Caesalpinia espinosa y 24 muestras para el Grupo Experimental 2: Hidróxido de Calcio + Gluconato de Clorhexidina al 2\%. 9 muestras para el Grupo Control Positivo: Amoxicilina + Ac. Clavulánico y 3 muestras para el Grupo Control Negativo: Suero Fisiológico. Posteriormente se rotuló las placas y se almacenó en la cámara de anaerobiosis a $37^{\circ} \mathrm{C}$ por 24 horas para proceder a tomar medidas, a las $24,48,72$ horas y 7 días.

Se realizó un análisis estadístico utilizando el programa SPSS versión 15.

\section{RESULTADOS}

Tabla $\mathbf{N}^{\circ} 1$. Efecto de la Caesalpinia espinosa al $60 \%$ en el halo inhibitorio del Enterococcus faecalis

\begin{tabular}{ccccc}
\hline Medición & \multicolumn{4}{c}{ Halo (mm) } \\
& Media & $\begin{array}{c}\text { Desviación } \\
\text { Estándar }\end{array}$ & Mínimo Máximo \\
\hline 24 horas & 9,03 & 0,38 & 8,50 & 9,75 \\
48 horas & 9,35 & 0,31 & 8,75 & 9,75 \\
72 horas & 9,77 & 0,31 & 9,25 & 10,25 \\
7 días & 9,89 & 0,27 & 9,50 & 10,25 \\
\hline
\end{tabular}

Fuente: Matriz de datos.

Según los resultados obtenidos (Tabla $\mathrm{N}^{\circ} 1$ ) podemos indicar que en el grupo experimental Caesalpinia espinosa la media del diámetro de la primera lectura es 9,03 , lo que indica que la Caesalpinia espinosa ha generado un espacio vacío (halo inhibitorio) de $9 \mathrm{~mm}$ en el cultivo microbiológico. Luego en la segunda lectura la medida fue de 9,35, en la tercera lectura la medida fue de $9,77 \mathrm{y}$ finalmente en la cuarta medida el valor fue de 9,89 , lo que indica que el diámetro va aumentando progresivamente. De lo cual se deduce que fue variable los valores máximos con tendencia a aumentar y luego a mantenerse igual entre el 3 día al día 7.

Tabla No 2. Efecto del Hidróxido de Calcio + Gluconato de Clorhexidina al 2\% en el halo inhibitorio del Enterococcus faecalis

\begin{tabular}{ccccc}
\hline Medición & \multicolumn{4}{c}{ Halo $(\mathrm{mm})$} \\
& Media & $\begin{array}{c}\text { Desviación } \\
\text { Estándar }\end{array}$ & \multicolumn{2}{c}{ Mínimo Máximo } \\
\hline 24 horas & 9,73 & 0,33 & 9,25 & 10,25 \\
48 horas & 9,62 & 0,25 & 9,25 & 10,00 \\
72 horas & 9,58 & 0,22 & 9,25 & 10,00 \\
7 días & 9,65 & 0,17 & 9,50 & 10,00 \\
\hline
\end{tabular}

Fuente: Matriz de datos. 


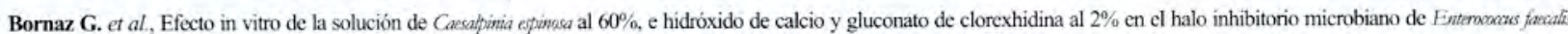

Según los resultados obtenidos (Tabla $\mathrm{N}^{\circ}$ 2) podemos afirmar que en el grupo experimental hidróxido de calcio + gluconato de clorhexidina, la media del diámetro de la primera lectura es 9,73 , lo que indica que el hidróxido de calcio + gluconato de clorhexidina ha generado un espacio vacío (halo inhibitorio) de casi $10 \mathrm{~mm}$ en el cultivo microbiológico.

De los datos podemos deducir que el diámetro del día 1 posee una ligera asimetría, posteriormente el día 2 se va tornando menos asimétrico, para luego el día 3 se va acercando más a una distribución normal, de igual forma que en el día 7 .

Tabla No 3. Análisis comparativo del halo inhibitorio a las 24 horas para Caesalpinia espinosa al $60 \%$ y el Hidróxido de Calcio + Gluconato de Clorhexidina al $2 \%$

\begin{tabular}{ccc}
\hline Halo (mm) & \multicolumn{2}{c}{$\begin{array}{c}\text { Grupo de Estudio } \\
\text { CA(OH) })_{2}^{+} \\
\text {Closhalpinia espinosa }\end{array}$} \\
\hline Media & 9,03 & 9,73 \\
Desviación & 0,38 & 0,33 \\
Mínimo & 8,50 & 9,25 \\
Máximo & 9,75 & 10,25 \\
Total & 24 & 24 \\
\hline
\end{tabular}

Fuente: Matriz de datos. $\mathrm{P}=0,000(\mathrm{P}<0,05)$ S.S.

Con respecto a los valores mostrados en la tabla $\mathrm{N}^{\circ} 3$, el promedio para el grupo experimental Caesalpinia espinosa es de 9,03 y para el grupo experimental Hidróxido de Calcio + Gluconato de Clorhexidina es de 9,73 lo cual determina que la diferencia entre los promedios de ambos halos inhibitorios fue de por lo menos $0,7 \mathrm{~mm}$, siendo el promedio de halos inhibitorios para grupo Hidróxido de Calcio + Gluconato de Clorhexidina mayor que el promedio de los halos inhibitorios del grupo Caesalpinia espinosa, la Desviación Estándar para el Grupo Caesalpinia espinosa es 0,38 y para el grupo Hidróxido de Calcio + Gluconato de Clorhexidina es de 0,33 , lo cual indica que los datos para el grupo de la Caesalpinia espinosa son relativamente más dispersos que el otro grupo experimental.

En conclusión, se observa que los promedios son diferentes $\mathrm{y}$ ambas distribuciones estadísticas tienen rangos variados para los valores obtenidos a las $24 \mathrm{hrs}$. Habiendo diferencia estadísticamente significativa entre los valores de ambos grupos.

Tabla $\mathrm{N}^{\circ} 4$. Análisis comparativo del halo inhibitorio a las 24 horas para Caesalpinia espinosa al $60 \%$ y la Amoxicilina + Ácido Clavulánico (control positivo)

\section{Halo $(\mathrm{mm}) \quad$ Grupo de Estudio}

\begin{tabular}{ccc} 
& Caesalpinia espinosa & $\begin{array}{c}\text { Amoxicilina - Ác. } \\
\text { Clavulánico }\end{array}$ \\
\hline Media & 9,03 & 16,22 \\
Desviación & 0,38 & 0,34 \\
Mínimo & 8,50 & 15,75 \\
Máximo & 9,75 & 16,75 \\
Total & 24 & 9 \\
\hline
\end{tabular}

Fuente: Matriz de datos. $\mathrm{P}=0,000(\mathrm{P}<0,05)$ S.S.
En la tabla $\mathrm{N}^{\circ} 4$, se muestran los valores promedio de halo inhibitorio para el grupo experimental Caesalpinia espinosa y para el grupo control, con una diferencia de 7,19 a favor del grupo control.

En resumen, se observa que los promedios son diferentes y ambas distribuciones estadísticas tienen rangos variados para los valores obtenidos a las $24 \mathrm{Hrs}$. Habiendo diferencia estadísticamente significativa entre los valores de ambos grupos.

Tabla $\mathbf{N}^{\circ}$ 5. Análisis comparativo del halo inhibitorio a los 7 días para Caesalpinia espinosa al $60 \%$ y el Hidróxido de Calcio + Gluconato de Clorhexidina al $2 \%$.

\begin{tabular}{|c|c|c|}
\hline \multirow[t]{2}{*}{ Halo (mm) } & \multicolumn{2}{|c|}{ Grupo de estudio } \\
\hline & Caesalpinia espinosa & $\underset{\text { Clorhexidina } 2 \%}{\mathrm{CA}(\mathrm{OH})_{2}+}$ \\
\hline Media & 9,89 & 9,65 \\
\hline Desviación & 0,27 & 0,17 \\
\hline Mínimo & 9,50 & 9,50 \\
\hline Máximo & 10,25 & 10,00 \\
\hline Total & 24 & 24 \\
\hline
\end{tabular}

Fuente: Matriz de datos. $\mathrm{P}=0,001(\mathrm{P}<0,05)$ S.S.

En la tabla 5, se observa que los promedios del halo inhibitorio en ambos grupos, son diferentes y ambas distribuciones estadísticas tienen rangos variados para los valores obtenidos el día 7. Habiendo diferencia estadísticamente significativa entre los valores de ambos grupos experimentales, nos permite concluir que el halo antimicrobiano fue mayor con el extracto de Caesalpinia espinosa al $60 \%$.

Tabla $\mathrm{N}^{\circ} 6$. Análisis comparativo del halo inhibitorio a los 7 días para Caesalpinia espinosa al $60 \%$ y la Amoxicilina + Ácido Clavulánico (control positivo).

\begin{tabular}{ccc}
\hline Halo (mm) & \multicolumn{2}{c}{$\begin{array}{c}\text { Grupo de estudio } \\
\text { Amoxicilina-Ác. } \\
\text { Clavulánico }\end{array}$} \\
\hline Caesalpinia espinosa & 9,89 & 17,66 \\
Desviación & 0,27 & 0,35 \\
Mínimo & 9,50 & 17,00 \\
Máximo & 10,25 & 18,25 \\
Total & 24 & 9 \\
\hline
\end{tabular}

Fuente: Matriz de datos. $\mathrm{P}=0,000(\mathrm{P}<0,05)$ S.S.

En la tabla $N^{\circ} 6$, los valores promedio para el grupo experimental Caesalpinia espinosa son de 9,89 y para el grupo control es de 17,66 lo cual determina que la diferencia entre los promedios de ambos halos inhibitorios fue de 7,77 $\mathrm{mm}$. En resumen, el promedio de halos inhibitorios para grupo control es mayor que el promedio de los halos inhibitorios del grupo Caesalpinia espinosa.

En conclusión se observa que los promedios son diferentes y ambas distribuciones estadísticas tienen rangos variados para los valores obtenidos el día 7 . Habiendo diferencia estadísticamente significativa entre los valores de ambos grupos. 
Bornaz G. et al., Efecto in vitro de la solución de Cassalpinia espinosa al 60\%, e hidróxido de calcio y gluconato de clorexhidina al $2 \%$ en el halo inhibitorio microbiano de Enéencacas faecales

\section{DISCUSIÓN}

Kloucek, P y col. (12), realizaron un ensayo antimicrobiano sobre extractos etanólicos al $80 \%$ de nueve plantas obtenidas por maceración durante 5 días, una de las muestras ensayadas fue la $C$. spinosa (vainas); se utilizaron cinco cepas Gram positivas y tres Gram negativas. Los resultados mostraron un Enterococcus faecalis en el que se observó una CIM de $0,5 \mathrm{mg} / \mathrm{ml}$, mientras que para Bacillus cereus fue de 8 , y de 16 para las otras bacterias, lo cual determina la especificidad y gran poder antimicrobiano de la tara frente a Enterococcus faecalis. Dicho resultado concuerda con nuestro estudio en el que también se observó una actividad antibacteriana notoria.

Liu H. y col. (13) realizaron un ensayo sobre la actividad antimicrobiano sobre la actividad antimicrobiana del extracto de Tara obtenido de las vainas, en el que determina que las vainas poseen mayor efecto antimicrobiano sobre cepas gram positivas que el extracto de las semillas. Esto concuerda con nuestro estudio debido a que el extracto que se preparó de las vainas de tara tuvo poder antimicrobiano sobre el Enterococcus faecalis que está considerado como un Gram Positivo.

Martínez L y col. (14) realizaron un ensayo sobre la actividad antibacteriana del Hidróxido de Calcio solo y con gluconato de Clorhexidina, comprobando la actividad antimicrobiana de dicha medicación a las 24,48 y 72 horas. El mismo que coincide con nuestro estudio donde también se comprobó el poder antimicrobiano en las tomas de medida.

\section{CONCLUSIONES}

PRIMERA: El efecto de la Caesalpinia espinosa al $60 \%$ en el halo inhibitorio del Enterococcus faecalis en promedio fue de $9,51 \mathrm{~mm}$ con una desviación estándar de 0,31 .

SEGUNDA: El efecto del Hidróxido de Calcio + Gluconato de clorhexidina $2 \%$ en el halo inhibitorio del Enterococcus faecalis en promedio fue de $9,64 \mathrm{~mm}$ con una desviación estándar de 0,24 .

TERCERA: Hubo diferencia significativa al comparar el efecto de la Caesalpinia espinosa al 60\% y el Hidróxido de Calcio + Gluconato de clorhexidina $2 \%$ en el halo inhibitorio del Enterococcus faecalis, esto con un margen de error del $5 \%$.

CUARTA: La hipótesis nula fue rechazada, debido a que el efecto antimicrobiano de la Caesalpinia espinosa al $60 \%$ fue mayor que el del Hidróxido de Calcio + Gluconato de clorhexidina $2 \%$ en el halo inhibitorio del Enterococcus faecalis, por lo tanto fue aceptada la hipótesis alterna.

\section{REFERENCIAS BIBLIOGRÁFICAS}

1. Gonzalez MA, Gonzalez N. Infecciones bacterianas de origen pulpar y periodontal. Oral Med Oral Pathol Oral Surg. 2004

2. Portenier I, Waltimo T. Haapasalo M. Enterococcus Fecalis and endash; the root canal survivor and "star" in post -treatment disease. Endod. Topics 2003;6:135-139

3. Stuart C. Schwartz S. Beeson T, Owatz C. Enterococcus faecalis: Its role in root canal treatment failure and current concepts in retreatment. J Endod. 2006;32 : 93 98

4. Attin et al, El hipoclorito de sodio disuelve tejido necrótico, no tejido vital. J International Endod. 2002

5. George S, Kishen A, Song K. The Role of Environmental Changes on Monospecies Biofilm Formation on Root Canal Wall by Enterococcus faecalis. J Endod. 2005; 31: 867-72.

6. Lima K, Fava L, Siqueira J. Susceptibilities of Enterococcus faecalis biofilms to some antimicrobial medications. J Endod. 2001;27: 616-9.

7. Barriga, C. Cultivo y Aprovechamiento de la Tara, Caesalpinia espinosa, en la Región Andina. Informe Técnico. Lima 2008

8. Mc Hugh C, Zhang P, Michalek S, Eleazer P. Ph required to kill Enterococcus faecalis in vitro. J Endod. 2004;30 : 218 219

9. Frais, S. Gulabivala K. Some factors affecting the concentration of avaible chlorine in commercial sources of sodium hypochlorite. Int Endod J. 2001

10. Espinel Pinzon M. Efecto antimicrobiano de hipoclorito de sodio y EDTA sobre Enterococcus faecalis. Tesis para optar el grado de magister en Salud Publica UPCSM, 2011.

11. Yolken R. Editores. Manual of Clinical Microbiology. American Society for Microbiology 2002.

12. Kloucek P, Lima K y Fava L. Evaluation of the antibacterial activities of calcium hydroxide, chlorhexidine, medicament. A Clinical and Laboratory Study. J Endodont, May, 2007.

13. Liu H, Zuckerman O, Weiss E, Mazor Y, Fuss Z. Antibacterial efficacy of a new chlorhexidine slow release device to disinfect dentinal tubules. J of Endod 2003 Jun.

14. Martinez L, Solak H, Oztan. The pH changes of four different calcium hydroxide mixtures used or for Intracanal medication. J of Oral Rehabilitation 2003.

\section{Correspondencia:}

Guillermo Bornaz Acosta: willy_bornaz@hotmail.com
Fecha de Recepción: 04/10/2014

Fecha de Aceptación: 03/12/2014 